\title{
Pneumatocele and cysts in a patient with severe acute respiratory syndrome coronavirus 2 infection
}

Nishal Brahmbhatt, MD, Omar Tamimi, MD, Henry Ellison, MD, Sai Katta, MD, Jihad Youssef, MD, Christopher Cortes, MD, and Deepa Gotur, MD, Houston, Tex

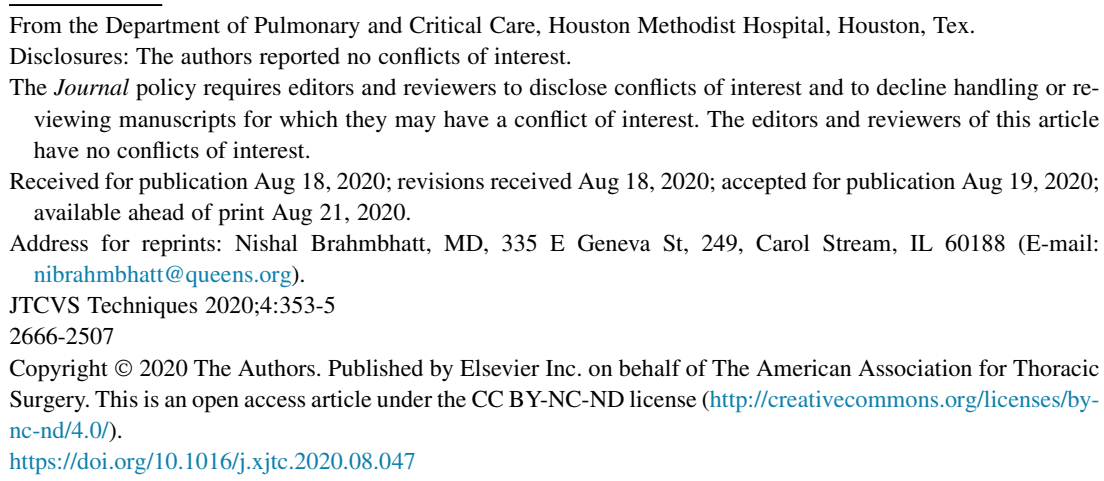

- Video clip is available online.

The year 2020 saw the introduction of a new health crisis brought on by the newly identified severe acute respiratory syndrome coronavirus 2 virus. As the world continued to understand this new disease process, its high infectious and mortality rate have been of much concern. Lung parenchymal changes associated this coronavirus disease 2019 (COVID-19) pneumonia have been described in the literature; however, the development of spontaneous pneumatocele has not been described. The aim of this article is to highlight one of the possible outcomes of acute respiratory distress syndrome (ARDS) associated with COVID-19 pneumonia or consequence of associated therapies.

A 66-year-old female patient with diabetes mellitus type II, hypertension, and hyperlipidemia presented with cough, shortness of breath, and fever for 2 weeks before presentation. She had no recent travel or interaction with sick contacts. She presented to an outside hospital and tested positive for COVID-19 by reverse transcription polymerase chain reaction on a nasopharyngeal specimen. Due to hypoxemia, she was admitted to the hospital and was initiated on hydroxychloroquine. Her oxygen requirement increased from $5 \mathrm{~L} /$ min to $15 \mathrm{~L} / \mathrm{min}$ oxygen flow by nasal cannula and was transferred to the intensive care unit for closer monitoring. She was transferred to our facility for greater level of care.

Upon arrival, oxygen support was optimized to high-flow oxygen at $40 \mathrm{~L} / \mathrm{min}$. Arterial blood gas revealed $\mathrm{pH} 7.48$, carbon dioxide tension 43, arterial oxygen tension $\left(\mathrm{PaO}_{2}\right)$ 74 , and $\mathrm{HCO}_{2}$ 32. Calculated $\mathrm{PaO}_{2}$ /inspired oxygen fraction release syndrome.

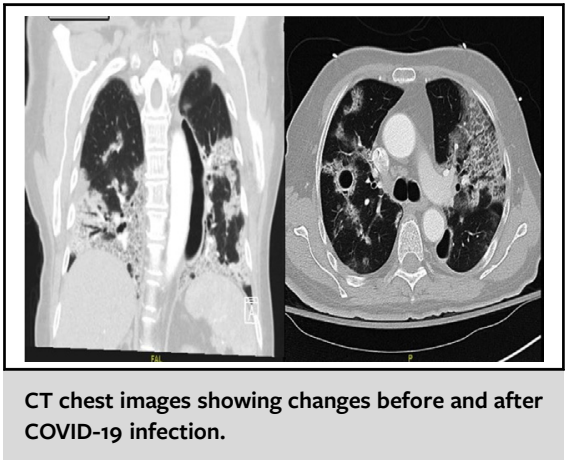

\begin{abstract}
CENTRAL MESSAGE
The changes in our patient's CT scan are a direct result of lung parenchymal destruction in setting of ARDS due to COVID-19 pneumonia. Aim is to create awareness and help the discussion in management.
\end{abstract}

See Commentaries on pages 356 and 358.

$\left(\mathrm{FIO}_{2}\right)$ ratio was 185 . Ferritin level was $824 \mathrm{ng} / \mathrm{mL}$, lactate dehydrogenase $265 \mathrm{U} / \mathrm{L}$, and C-reactive protein level was $21.25 \mathrm{mg} / \mathrm{dL}$. D-dimer level was $1.86 \mu \mathrm{g} / \mathrm{mL}$. She received convalescent plasma as well as tocilizumab for cytokine

One week after admission, she developed chest pain and became increasingly hypoxemic, requiring increasing oxygen support of $50 \mathrm{~L} / \mathrm{min}$ and an $\mathrm{FIO}_{2} 60 \%$ on high-flow nasal cannula (HFNC). Arterial blood gas revealed $\mathrm{pH}$ 7.44, carbon dioxide tension $42, \mathrm{PaO}_{2} 74, \mathrm{HCO}_{3} 28.2$, and $\mathrm{PaO}_{2} / \mathrm{FIO}_{2}$ ratio 123 . Chest radiograph revealed a lucency on the left lung lateral to the aorta (Figure 1). To further characterize this lucency and evaluate for pulmonary embolism, a computed tomography (CT) scan of the chest, pulmonary embolism protocol, was performed and was remarkable for changes consistent with COVID-19 pneumonia. A pneumatocele in the left hemithorax and a thinwalled cyst were noted in the right hemithorax (Figure 2). A thorough review of images did not reveal bronchopleural or esophageal-pleural fistula. 

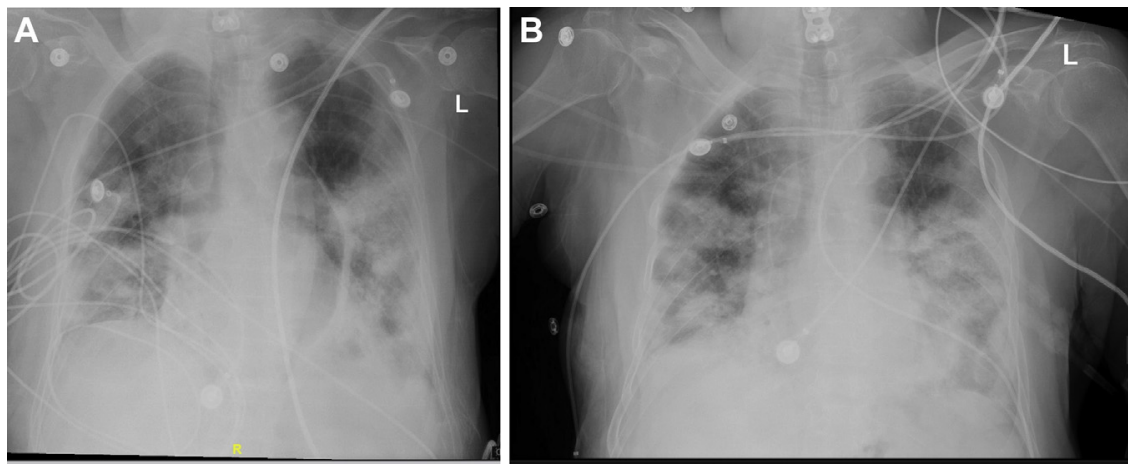

FIGURE 1. A, AP chest film on day 4. Patient was noted to develop worsening chest pressure and hypoxemia. Black arrows highlight a 1.5 -cm $\times 0.3-\mathrm{cm}$ lucency over the left mediastinum suggestive of a pneumatocele with underlying bilateral patchy consolidation. B, AP chest film showing resolution of pneumatocele with persistent consolidative changes.

The patient did not have a previous CT scan of the chest for comparison. A review of the bases of the lung on a CT of the abdomen done 3 months before admission revealed no abnormalities. No previous history of lung disease or tobacco use was noted. HIV was negative and alpha-1-antitrypsin levels were $124 \mathrm{mg} / \mathrm{dL}$. A pig-tail catheter was inserted using CT guidance. Pleural fluid analysis was exudative by modified Light's criteria (Video 1 and Table 1). Cultures from pleural fluid, blood, and sputum were negative. Follow-up radiograph of the chest revealed resolution of the pneumatocele. After a 15-day hospital stay, she improved and was discharged home.
This was a retrospective chart review. Attempts were made to contact patient for consent; however, she was not reachable.

\section{DISCUSSION}

We present a case of a 66-year-old female patient with ARDS from COVID-19 pneumonia who developed cysts and pneumatocele while on HFNC. Blood, pleural fluid, and sputum cultures were negative. Asymptomatic pneumatocele should be conservatively managed with close observation, given concerns for intervention associated complications such as bronchopleural fistulae. A pig-tail catheter was inserted to evacuate the air and effusion,
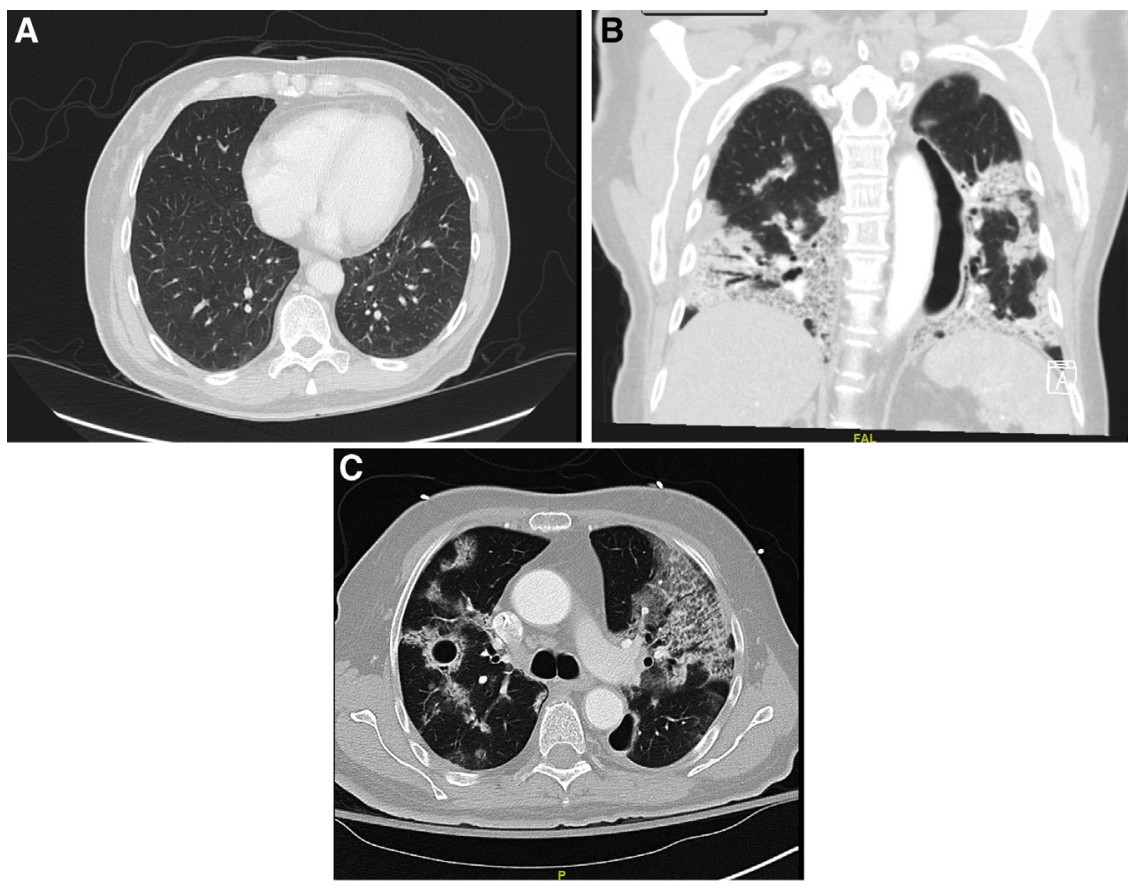

FIGURE 2. A, Baseline computed tomography with basal lung cuts showing normal lung parenchyma. B, Coronal section showing the pneumatocele. A complete rind is seen around the pneumatocele. Bilateral patchy consolidative changes noted. C, Cross-sectional image showing thin walled cyst on right, pneumatocele on left. 


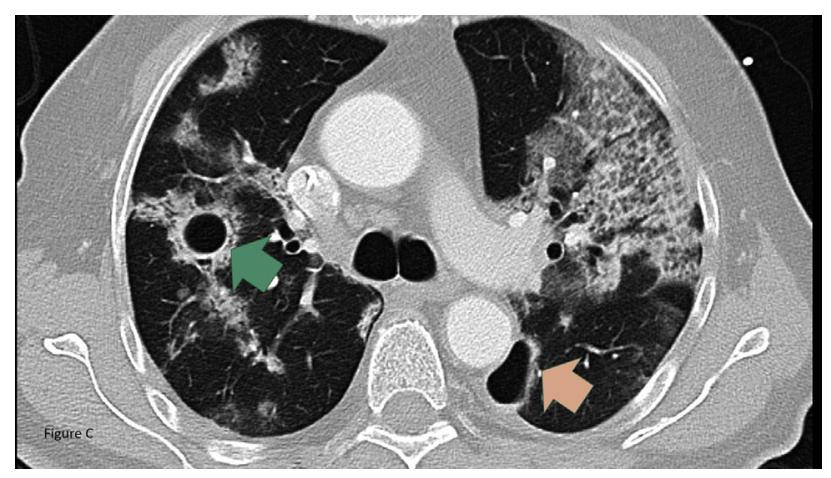

VIDEO 1. An overview of the case report with descriptive analysis of chest imaging. Video available at: https://www.jtcvs.org/article/S26662507(20)30426-0/fulltext.

alleviate symptoms of chest pressure, improve hypoxemia, and evaluate for infection. There was no concern for tension pneumothorax or sudden death.

Radiologic findings of COVID-19 pneumonia have been described as diffuse bilateral peripheral ground-glass opacities with or without consolidations. ${ }^{1} \mathrm{Ng}$ and colleagues ${ }^{1}$ noted that the bilateral upper and lower lobes were more affected than the right middle lobe and lingula. In a review, Desai and colleagues ${ }^{2}$ reported that in the early phase of ARDS, ground-glass opacities and consolidations were seen in $85 \%$ of patients. Ground-glass opacities were noted to be in nondependent positions, whereas consolidations were seen in dependent positions. In the late phase of ARDS, a majority of patients developed changes consistent with pulmonary fibrosis, described as thick, interlobulated septae and parenchymal bands. Honeycombing and cyst development were seen in severe cases. ${ }^{3}$

Although ARDS may likely explain the findings seen in our patient, we cannot safely rule out the possibility of pneumatocele being formed as a direct consequence of tocilizumab administration. Tocilizumab is a human interleukin-6 monoclonal antibody associated with increased risk of bowel perforation. ${ }^{4}$

Barotrauma in mechanically ventilated patients is a well described and presents as a pneumothorax or pneumomediastinum. While documented, barotrauma
TABLE 1. Pleural fluid analysis

\begin{tabular}{ll}
\hline \multicolumn{1}{c}{ Chemistry } & \multicolumn{1}{c}{ Hematology } \\
\hline Albumin: $1.7 \mathrm{~g} / \mathrm{dL}$ & Appearance: bloody \\
Amylase: $24 \mathrm{U} / \mathrm{L}$ & Color: red \\
\hline Glucose: $158 \mathrm{mg} / \mathrm{dL}$ & Eosinophils: $2 \%$ \\
\hline LDH: $472 \mathrm{U} / \mathrm{L}$ & RBCs: $505,000 / \mathrm{cm}^{3}$ \\
\hline pH: 8 & Lymphocytes: $7 \%$ \\
\hline Protein: $3.8 \mathrm{~g} / \mathrm{dL}$ & Macrophages: $12 \%$ \\
& Mesothelial cells: $2 \%$ \\
\hline & Neutrophils: $77 \%$ \\
\hline & Nucleated cells: $9946 / \mathrm{cm}^{3}$ \\
\hline
\end{tabular}

$L D H$, Lactate dehydrogenase; $R B C$, red blood cell.

associated with the use of HFNC is rare. ${ }^{5}$ Given the rarity of barotrauma associated with HFNC, it is less likely that the changes seen in our patient are a result of barotrauma. The changes noted in our patient's CT scan are a direct result of lung parenchymal destruction in the setting of ARDS secondary to COVID-19 pneumonia progression to fibrosis, cyst, and pneumatocele formation.

We highlight the rapid progression of severe destructive changes to otherwise-normal healthy lung parenchyma in the setting of COVID-19 pneumonia over a period of 4 weeks in a patient who did not require mechanical ventilation.

\section{References}

1. Ng MY, Lee E, Yang J, Yang F, Li X, Wang H, et al. Imaging profile of the COVID 19 infection: radiologic findings and literature review. Radiol Cardiothorac Imaging. 2020;2:e200034.

2. Desai SR, Wells AU, Rubens MB, Evans TW, Hansell DM. Acute respiratory distress syndrome: CT abnormalities at long-term follow-up. Radiology. 1999; 210:29-35.

3. Chiumello D, Coppola S, Froio S, Gotti M. What's next after ARDS: long term outcomes. Respir Care. 2016;61:689-99.

4. Singh JA, Beg S, Lopez-Olivo MA. Tocilizumab for rheumatoid arthritis Cochrane Database Syst Rev. 2010;7:CD008331.

5. Piastra M, Morena TC, Antonelli M, Conti G. Uncommon barotrauma while on high-flow nasal cannula. Intensive Care Med. 2018;44:2288-9. 